\title{
Características do processo de ingestão de forragem por novilhas holandesas em pastagens de capim-mombaça ${ }^{1}$
}

\section{Ana Luisa Palhano2 ${ }^{2}$, Paulo César de Faccio Carvalho ${ }^{3}$, João Ricardo Dittrich ${ }^{4}$, Aníbal de Moraes $^{4}$, Sila Carneiro da Silva ${ }^{5}$, Alda Lúcia Gomes Monteiro ${ }^{4}$}

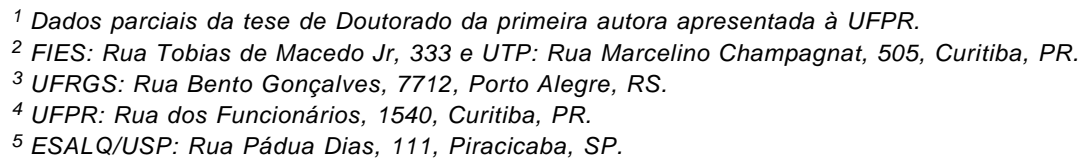

RESUMO - Para avaliação da influência da estrutura do dossel de pastagens tropicais sobre o processo de ingestão de forragem por bovinos, cinco alturas de dossel (60, 80, 100, 120 e $140 \mathrm{~cm}$ ) de uma pastagem de capim-mombaça (Panicum maximum, Jacq.) foram estabelecidas, em um delineamento inteiramente casualizado, com duas repetições. Quatro novilhas da raça Holandês Preto e Branco com coletores de fezes e urina foram utilizadas em testes de pastejo de 45 minutos. O número de bocados e o tempo de alimentação foram registrados com o uso de contadores e cronômetros. A massa de forragem total consumida foi obtida pela técnica de dupla pesagem. Com o aumento na altura do dossel, observou-se aumento linear na massa de bocado. O aumento do comprimento das lâminas foliares expandidas e em expansão resultou em consumo quase que individual de folhas, refletindo em aumento linear no tempo por bocado e em redução linear na taxa de bocados. Uma vez que o aumento no tempo por bocado foi proporcionalmente maior que o aumento da massa de bocado, a massa de forragem total consumida e a taxa de ingestão estabilizaram-se nas maiores alturas da pastagem. As limitações físicas impostas pela estrutura do dossel levaram a menor eficiência de captura de forragem pelos animais nas maiores alturas do dossel. O consumo de forragem foi maximizado à altura de $109,3 \mathrm{~cm}$.

Palavras-chave: bovinos, estrutura do dossel, massa de bocado, pastagem tropical

\section{Forage intake characteristics on mombaçagrass pastures grazed by Holstein heifers}

\begin{abstract}
In order to evaluate the influence of tropical grass sward structure on the intake of grazing cattle, five sward heights $(60,80,100,120$, and $140 \mathrm{~cm})$ of Panicum maximum Jacq. were established, in a completely randomized design with two replications. Four black-and-white coat Holstein heifers, carrying feces and urine bags, were used in grazing tests of 45 minutes each. Number of bites and feeding time were registered by counters and chronometers. Total herbage mass consumed was obtained by double sampling technique. Increasing sward height increased bite mass, followed by an increase in chewing number. The increase in the length of both expanded leaves and expanding leaves, resulted in individual bite per leaf, leading to an increase in biting time, and consequent linear decrease in bite rate. Since increases in time per bite was proportionally greater than that for bite mass, total herbage mass consumed and intake rate stabilized at the highest sward heights. Under these conditions, the physical limitation imposed by sward structure led to a lower efficiency of foraging by the animals, even at high forage allowance situation. Intake rate was maximized at pasture height of $109.3 \mathrm{~cm}$.
\end{abstract}

Key Words: bovine, bite mass, sward structure, tropical grass

\section{Introdução}

O consumo diário de forragem é o aspecto central para maior compreensão do comportamento dos animais em pastejo, diretamente influenciado por fatores relacionados à planta forrageira e ao animal. O baixo consumo de forragem tem sido apontado como uma das principais limitações ao desempenho animal em regiões tropicais, parte relacionada ao efeito climático sobre a qualidade da forragem (Van Soest, 1996) e parte às características estruturais das espécies forrageiras tropicais (Carvalho et al., 2001a).

A estrutura do dossel forrageiro, relacionada à distribuição vertical e horizontal de componentes morfológicos e botânicos (Cangiano et al, 2002), pode influenciar a facilidade de apreensão da forragem pelos animais (Stobbs, 1973a,b; Nabinger \& Pontes, 2001) e exercer efeitos sobre seu consumo diário. 
Assim, a massa do bocado, principal estratégia dos animais para regular a ingestão diária de nutrientes, é a base de um ajuste feito pelos animais entre destinar tempo para mastigação, o que pode aumentar a taxa de digestão e passagem, ou para apreensão de novos bocados visando manter o nível de consumo (Prache \& Peyraud, 2001).

Em condições de baixa oferta de forragem, o animal tende a aumentar o tempo de pastejo e a freqüência de bocados (Gordon \& Lascano, 1993) para atender sua demanda diária de ingestão de MS. Esses ajustes são recursos importantes do animal em pastejo para o atendimento de suas exigências nutricionais diárias (Tharmaraj et al.,2003), porém, com certas limitações (Hodgson, 1990).

Por outro lado, segundo Carvalho et al. (2001a), em condições de elevada oferta de forragem, a ingestão de forragem pelos animais também pode ser restringida. Esses autores observaram redução na taxa de consumo por ovinos como conseqüência do maior tempo para formação do bocado, ocasionado pela disposição esparsa das folhas de capim-tanzânia (P. maximum, (L.) Leeke), espécie tropical de hábito de crescimento cespitoso.

Objetivou-se neste estudo avaliar a influência da estrutura do dossel em pastagens de capim-mombaça sobre os aspectos de ingestão de forragem de novilhas leiteiras em pastejo.

\section{Material e Métodos}

Foram testadas cinco alturas de dossel $(60,80,100,120$ e $140 \mathrm{~cm}$ ) em uma pastagem de capim-mombaça (Panicum maximum (L.) Leeke), com duas repetições, estabelecidos em piquetes de $540 \mathrm{~m}^{2}$. No período de fevereiro a abril de 2002 , foram realizados dez testes de pastejo, de acordo com a altura do dossel proposta para cada tratamento, de modo que as plantas permaneceram em crescimento livre desde o corte de igualação ocorrido em dezembro de 2001. Durante os testes, foram utilizadas quatro novilhas holandesas preto e brancas (peso médio de $150 \mathrm{~kg}$ ) portando coletores de fezes e urina.

Em cada dia de avaliação, foram feitos os procedimentos de caracterização da estrutura do dossel e os testes de pastejo para coleta dos dados relacionados à ingestão de forragem. A altura da pastagem foi medida antes e após os testes de pastejo utilizando-se uma régua graduada denominada sward stick(Barthram, 1985). Para quantificação da massa de forragem, foram coletadas cinco amostras da biomassa vegetal aérea de cada repetição, utilizando-se um equipamento denominado estratificador, com área basal de $0,5625 \mathrm{~m}^{2}$, segmentado a cada $20 \mathrm{~cm}$ de altura. O material colhido foi separado nas frações lâminas foliares, colmos + bainhas e material senescente. A disponibilidade total de forragem e de seus componentes foi determinada pela soma dos sucessivos estratos colhidos em cada amostragem, utilizados também para o cálculo das respectivas densidades volumétricas.

Os teores de PB, FDN e FDA da forragem disponível foram determinados utilizando-se, respectivamente, os métodos Kjeldhal e Detergente. O material analisado foi o mesmo colhido para quantificação da massa total de forragem; no entanto, avaliaram-se apenas os estratos superiores a 50\% da altura proposta, separados nas frações lâminas foliares e colmos + bainhas.

Para medição do comprimento de lâminas foliares expandidas e em expansão, foram utilizados 100 perfilhos marcados com fio de telefone nos quais foram medidas as folhas expandidas, desde a lígula até seu ápice, e em expansão, desde a lígula da penúltima folha expandida (abaixo dela) até seu ápice.

Os testes de pastejo foram conduzidos segundo metodologia da dupla pesagem, proposta por Penning \& Hooper (1985). Após jejum de sólidos e líquidos (6 horas) no início da manhã, os animais experimentais foram preparados com os coletores de fezes e de urina e, em seguida, foram pesados e encaminhados aos piquetes experimentais juntamente com três animais acompanhantes. A inclusão de animais acompanhantes visou à obtenção do efeito de grupo.

Os animais experimentais foram divididos em dois grupos: o primeiro (animais A e B) permaneceu amarrado próximo aos piquetes e impossibilitado de consumir água e alimentos para avaliação das perdas metabólicas, determinadas mediante a variação de peso durante o período, e o segundo grupo (animais C e D) foi avaliado por meio de teste de pastejo, com duração de 45 minutos, sendo monitorados por quatro avaliadores que trabalharam em duplas, cada um avaliando um animal na pastagem e registrando o número de bocados e o tempo de alimentação com auxílio de contadores e cronômetros.

Paralelamente aos testes, foram retiradas manualmente, simulando o pastejo, conforme metodologia denominada hand plucking, 20 amostras de forragem em cada sessão de pastejo (Burns et al., 1989), com o objetivo de quantificar o teor de MS da forragem selecionada e ingerida pelos animais.

Ao final dos 45 minutos, os animais $\mathrm{C}$ e $\mathrm{D}$ foram pesados (portando os coletores de fezes e urina) para obtenção da diferença de peso entre a entrada e saída da pastagem. Os animais A e B, que aguardavam, foram também pesados e encaminhados à segunda sessão de pastejo, quando foram repetidos os mesmos procedimentos enquanto os animais $\mathrm{C}$ e D ficaram amarrados. Ao fim do teste, os animais foram encaminhados às áreas adicionais com pastagem da mesma espécie forrageira. 
O curto período de condução dos testes foi estabelecido para que não houvesse interferência de processos digestivos sobre a dinâmica de ingestão de forragem.

Para avaliação do comportamento ingestivo dos animais, as seguintes relações foram calculadas: taxa de bocados (bocados por minuto); tempo por bocado (segundos por bocado); massa de forragem total consumida ( $\mathrm{g}$ de $\mathrm{MS} / \mathrm{kg}$ de $\mathrm{PV}$ ); massa do bocado (mg de MS/bocado/kg de PV); taxa de ingestão (g MS/minuto/kg de PV). Os valores de massa de forragem total consumida, massa de bocado e taxa de ingestão foram expressos em relação ao peso dos animais.

O cálculo da massa de forragem total consumida foi feito utilizando-se a diferença de peso de cada animal entre as duas pesagens, adicionado de sua respectiva perda metabólica, obtendo-se o valor original corrigido para MS. A massa de bocado foi calculada pela relação entre a massa de forragem total consumida e o número de bocados registrado durante cada teste de pastejo.

O delineamento estatístico utilizado foi o inteiramente ao acaso, com cinco tratamentos e duas repetições e os dados obtidos foram analisados por meio de regressões polinomiais de primeiro e segundo graus, utilizando-se o aplicativo STATGRAPHICS PLUS, versão 4.1. Para os testes de médias, aplicou-se o teste de Duncan, a 5\% de significância.

\section{Resultados e Discussão}

Na Tabela 1 são apresentadas as características estruturais do dossel forrageiro do capim-mombaça nas diferentes alturas avaliadas.
Os valores de oferta de forragem - expressos pela razão volume de MS e unidades animais presentes, utilizados para dimensionar e verificar o grau de eficiência de sistemas rotativos - situaram-se na amplitude de 300 a $600 \mathrm{~m}^{3} / \mathrm{UA}$, considerada adequada e indicativa de sistemas de manejo rotativo adequadamente conduzidos (Duru et al., 2000).

Os valores de massa de forragem e de lâminas foliares aumentaram linearmente (Figura 1) com o incremento na altura do dossel, que variou de 7.500 a $17.000 \mathrm{~kg} / \mathrm{ha}$, refletindo o efeito da altura de manejo sobre a disponibilidade de forragem (Penning et al., 1991).

A densidade volumétrica da forragem e de colmos + bainhas (média dos estratos) não foi influenciada pelo aumento da altura do dossel (Tabela 1), apesar do incremento linear da MS de forragem. Esse resultado provavelmente está relacionado ao efeito do aumento da altura, que diluiu a MS total produzida ao longo de seus estratos. A densidade volumétrica de lâminas aumentou apenas até a altura de $100 \mathrm{~cm}$ e em seguida estabilizou.

Quando considerada ao longo dos estratos amostrados, a densidade de forragem reduziu com o aumento da distância ao solo (Tabela 2), estrutura característica de gramíneas forrageiras tropicais.

Os teores de PB da fração lâminas foliares referentes ao estrato médio superior do dossel reduziram de 20,5 para $9,7 \%$ com o aumento em altura do dossel, enquanto os teores de FDN e FDA apresentaram tendência inversa. Esses resultados se devem ao acúmulo progressivamente menor de nitrogênio em relação ao carbono com o crescimento da planta. O aumento no comprimento das lâminas foliares exige maior proporção

Tabela 1 - Alturas efetivas do dossel na entrada e saída dos animais da pastagem; oferta de forragem; densidade volumétrica de forragem, de colmos + bainhas e de lâminas foliares; massa de forragem e de lâminas foliares e teores de PB, FDN, FDA e MS do capim-mombaça

Table 1 - Effective sward heights at the beginning and at the end of the grazing tests, herbage allowance, leaf blade allowance, herbage, stem plus leaf sheath, and leaf blade volumetric density, herbage and leaves mass, PB, NDF, ADF and DM of mombaçagrass

\begin{tabular}{|c|c|c|c|c|c|c|}
\hline \multirow[t]{2}{*}{$\begin{array}{l}\text { Característica estrutural do dossel } \\
\text { Sward structural characteristic }\end{array}$} & \multicolumn{5}{|c|}{$\begin{array}{l}\text { Altura do dossel }(\mathrm{cm}) \\
\text { Sward height }\end{array}$} & \multirow[t]{2}{*}{$\mathrm{CV}(\%)$} \\
\hline & 60 & 80 & 100 & 120 & 140 & \\
\hline Altura real de entrada $(\mathrm{cm})$ (Pre-grazing sward height, $\mathrm{cm})$ & $58,9 \mathrm{e}$ & $79,3 \mathrm{~d}$ & $96,5 \mathrm{c}$ & $116,6 b$ & $133,0 \mathrm{a}$ & 2,5 \\
\hline Altural real de saída $(\mathrm{cm})$ (Post-grazing sward height, $\mathrm{cm})$ & $42,7 \mathrm{~d}$ & $59,6 \mathrm{c}$ & $96,4 b$ & $105,3 \mathrm{ab}$ & $114,1 \mathrm{a}$ & 4,8 \\
\hline Oferta de forragem $\left(\mathrm{m}^{3} / \mathrm{UA}\right)$ (Forage allowance, $\left.\mathrm{m}^{3} / U A\right)$ & $237 \mathrm{~d}$ & $293 c$ & $334 b$ & $410 \mathrm{a}$ & $431 \mathrm{a}$ & 3,4 \\
\hline Oferta de forragem (kg MS/100 kg PV) (Forage allowance, $\mathrm{kg} \mathrm{DM/100} \mathrm{kg} \mathrm{LW}$ ) & $59,4 b$ & $66,8 \mathrm{ab}$ & $76,6 \mathrm{ab}$ & $89,0 \mathrm{ab}$ & $104,4 \mathrm{a}$ & 14,4 \\
\hline Oferta de lâminas (kg MS/100 kg PV) (Leaf blade allowance, $\mathrm{kg} \mathrm{DM/100} \mathrm{kg} \mathrm{LW)}$ & $9,9 \mathrm{c}$ & $21,1 \mathrm{bc}$ & $28,8 \mathrm{~b}$ & $35,7 \mathrm{ab}$ & $46,5 \mathrm{a}$ & 20,3 \\
\hline Densidade de forragem $\left(\mathrm{kg} \mathrm{MS} / \mathrm{m}^{3}\right)$ (Forage density, $\mathrm{kg} \mathrm{DM} / \mathrm{m}^{3}$ ) & $1,26 \mathrm{a}$ & $1,14 \mathrm{a}$ & $1,10 \mathrm{a}$ & $1,09 \mathrm{a}$ & $1,22 \mathrm{a}$ & 15,8 \\
\hline Densidade de colmos $\left(\mathrm{kg} \mathrm{MS} / \mathrm{m}^{-3}\right)$ (Stem density, $\mathrm{kg} \mathrm{DM} / \mathrm{m}^{3}$ ) & $0,45 \mathrm{a}$ & $0,34 \mathrm{a}$ & $0,33 \mathrm{a}$ & $0,41 \mathrm{a}$ & $0,41 \mathrm{a}$ & 25,2 \\
\hline Densidade de lâminas $\left(\mathrm{kg} \mathrm{MS} / \mathrm{m}^{-3}\right.$ ) (Leaf blade density, $\mathrm{kg} \mathrm{DM} / \mathrm{m}^{3}$ ) & $0,21 b$ & $0,36 \mathrm{ab}$ & $0,43 \mathrm{a}$ & $0,44 \mathrm{a}$ & $0,54 \mathrm{a}$ & 19,0 \\
\hline Massa de forragem (kg MS/ha) (Forage mass, $\mathrm{kg} \mathrm{DM/ha)}$ & $7.569 \mathrm{c}$ & $9.130 \mathrm{bc}$ & $11.063 \mathrm{bc}$ & $13.126 \mathrm{ab}$ & $17.247 \mathrm{a}$ & 13,2 \\
\hline Massa de lâminas (kg MS/ha) (Leaf blade mass, $\mathrm{kg} \mathrm{DM/ha)}$ & $1.399 \mathrm{~d}$ & $2.911 \mathrm{~cd}$ & $4.114 \mathrm{bc}$ & $5.328 b$ & $7.678 \mathrm{a}$ & 17,1 \\
\hline $\mathrm{PB}(\%)(C P, \%)$ & $19,2^{\mathrm{a}}$ & $16,1 \mathrm{a}$ & $11,7 \mathrm{~b}$ & $12,2 b$ & $10,1 \mathrm{~b}$ & 10,0 \\
\hline FDN $(\%)(N D F, \%)$ & $66,2^{\mathrm{a}}$ & $64,7 \mathrm{a}$ & $68,4^{\mathrm{a}}$ & $74,4 \mathrm{a}$ & $72,6 a$ & 15,6 \\
\hline $\operatorname{MS}(\%)(D M, \%)$ & $16,9^{\mathrm{a}}$ & $23,1 b$ & $27,1 \mathrm{bc}$ & $27,0 \mathrm{bc}$ & $33,0 \mathrm{c}$ & 9,3 \\
\hline
\end{tabular}

Médias seguidas da mesma letra na mesma linha não diferem $(P<0,05)$ pelo teste Duncan.

Means followed by the same letter do not differ $(P<0.05)$ by Duncan test. 
a
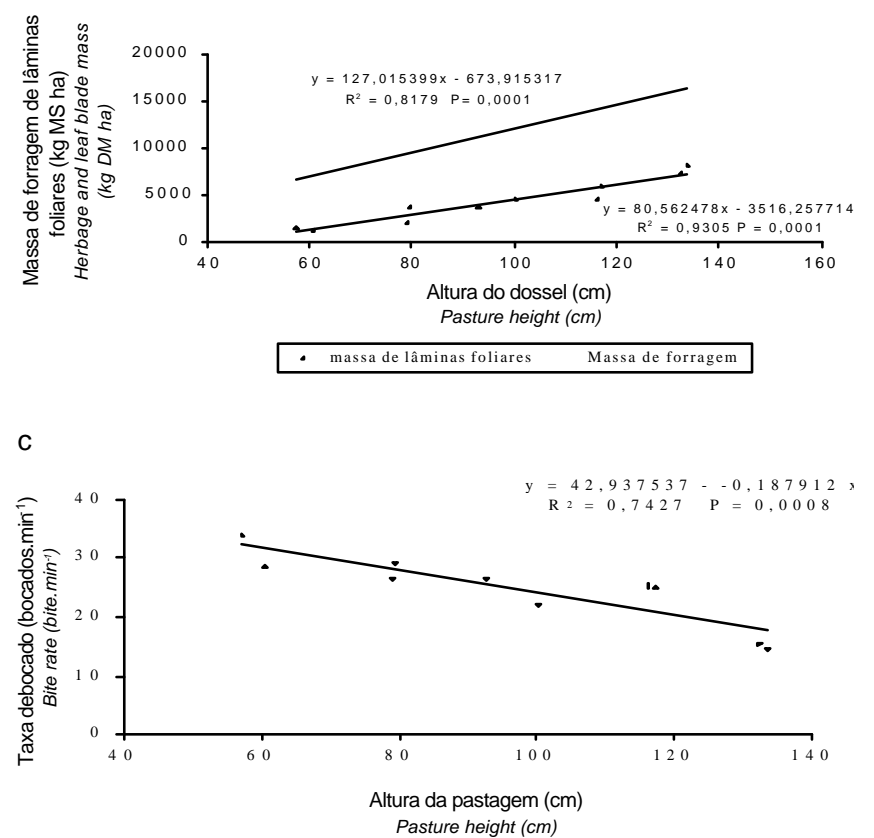

b

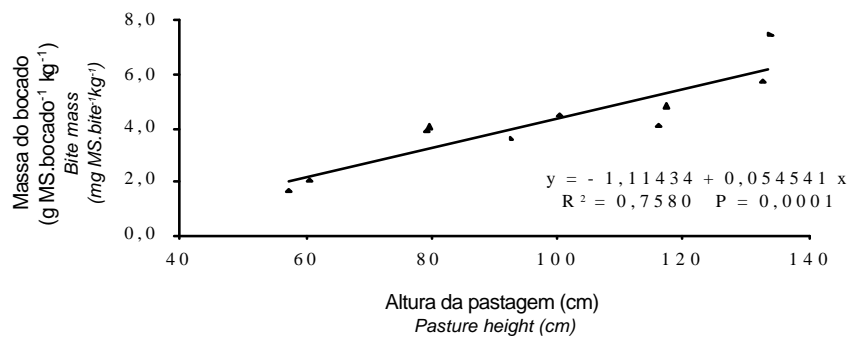

d

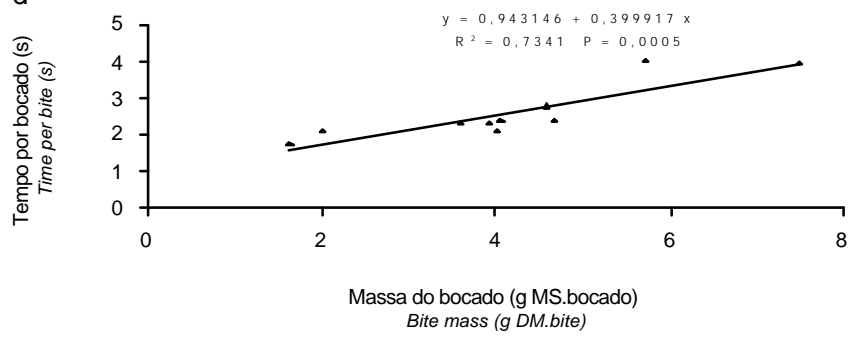

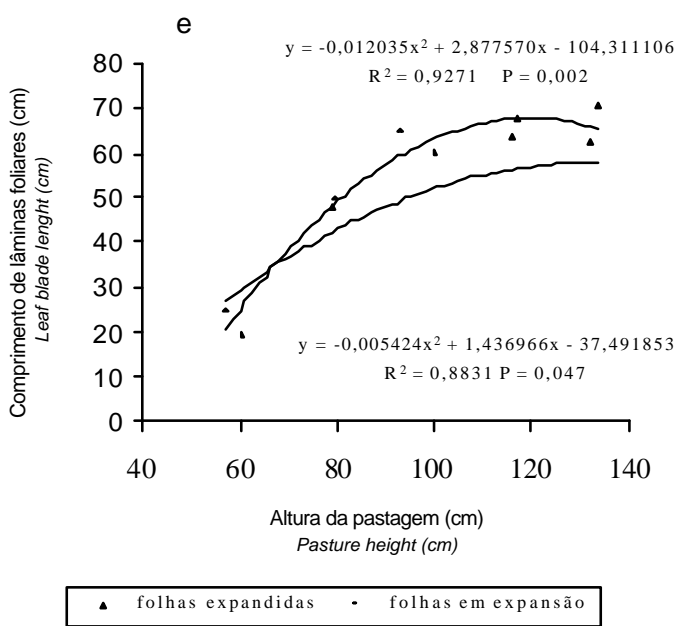

Figura 1 - Relação entre altura do dossel e (a) massa de forragem e de lâminas foliares (kg MS.ha ${ }^{-1}$ ), b) massa do bocado ( $\mathrm{g} \mathrm{MS}$. bocado $^{-1} .100$ $\mathrm{kg} \mathrm{PV}^{-1}$ ), c) taxa de bocados (bocados. $\mathrm{s}^{-1}$ ), d) tempo por bocado(s) e e) comprimento de lâminas foliares(cm) de capim-mombaça.

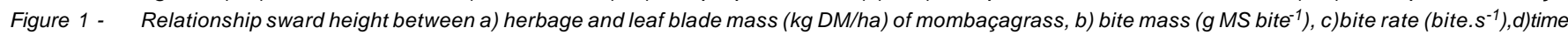
per bite $(\mathrm{s})$ and $\mathrm{e}$ ) leaf blades length $(\mathrm{cm})$ of mombaçagrass.

de tecidos estruturais, principalmente da nervura central da folha, para manter sua sustentação e otimizar a captação de luz (Lemaire \& Gastal, 1997). Esses tecidos, constituídos principalmente de feixes vasculares e esclerênquima, apresentam baixo teor de nitrogênio e altos valores de fibra.

A massa do bocado aumentou linearmente com o aumento da altura do dossel (Figura 1b), o que está de acordo com os resultados descritos por Cangiano et al. (2002), Damasceno et al. (2003 b) e Tharmaraj et al. (2003).

A relação positiva entre altura do dossel/massa de forragem disponível e massa de bocado/consumo de forra gem é conhecida como resposta funcional (Hodgson, 1990) e pode ser descrita como uma função curvilinear (Carvalho et al., 2001b), significando que pastagens mantidas mais altas potencializam a profundidade do bocado, determinante da massa do bocado e, conseqüentemente, do consumo de forragem (Carvalho, 1997). O aumento linear no teor de MS da forragem ingerida em cada bocado com a elevação em altura do dossel contribuiu também para maior massa de bocado (Tabela 1), indicando que esse aumento da massa de bocado não decorre apenas da geometria, ou seja, da profundidade e da área do bocado, como descrito na litera tura 
Tabela 2 - Densidade volumétrica de forragem de capimmombaça $\left(\mathrm{kg} \mathrm{MS} / \mathrm{m}^{3}\right)$ manejado em diferentes alturas de dossel

Table 2 - Herbage volumetric density of mombaçagrass pastures ( $\mathrm{kg}$ DM $\mathrm{m}^{3}$ ), according to different sward heights

\begin{tabular}{|c|c|c|c|c|c|}
\hline & \multicolumn{5}{|c|}{$\begin{array}{l}\text { Altura do dossel }(\mathrm{cm}) \\
\text { Sward height }\end{array}$} \\
\hline & 60 & 80 & 100 & 120 & 140 \\
\hline \multicolumn{2}{|l|}{ Estrato do dossel $(\mathrm{cm})$} & \multicolumn{3}{|c|}{$\left(\mathrm{kg}\right.$ de $\left.\mathrm{MS} / \mathrm{m}^{3}\right)$} & \\
\hline \multicolumn{2}{|l|}{ Sward strata } & \multicolumn{3}{|c|}{$\left(\mathrm{kgDM} / \mathrm{m}^{3}\right)$} & \\
\hline $120-140$ & & & & & 0,14 \\
\hline $100-120$ & & & & 0,10 & 0,32 \\
\hline $80-100$ & & & 0,15 & 0,27 & 0,63 \\
\hline $60-80$ & & 0,05 & 0,47 & 0,62 & 0,97 \\
\hline $40-60$ & 0,16 & 0,38 & 0,82 & 1,08 & 1,61 \\
\hline $20-40$ & 0,69 & 1,05 & 1,40 & 1,46 & 1,85 \\
\hline $0-20$ & 2,92 & 3,08 & 2,66 & 2,98 & 3,02 \\
\hline $\mathrm{CV}(\%)$ & \multicolumn{5}{|c|}{$\begin{array}{c}\text { Média do perfil }\left(\mathrm{kg} \mathrm{MS} / \mathrm{m}^{3}\right) \\
\text { Profile average }\left(\mathrm{kg} \mathrm{DM} / \mathrm{m}^{3}\right)\end{array}$} \\
\hline 15,8 & $1,26 a^{*}$ & $1,14 \mathrm{a}$ & $1,10 \mathrm{a}$ & $1,09 \mathrm{a}$ & $1,22 \mathrm{a}$ \\
\hline
\end{tabular}

Médias seguidas da mesma letra não diferem pelo teste Duncan a $5 \%$. Means followed by the same letter do not differ by Duncan test at $5 \%$.

(Ungar, 1996), mas também da alteração da concentração de massa por unidade de superfície de folha, hipótese registrada por Castro (2002).

Os valores encontrados para a massa do bocado variaram de 0,202 a 0,747 g MS/bocado/animal. Em estudo com animais jovens, Demment \& Laca (1993) verificaram valores de 0,5 a 3,0 g e Cangiano et al (2002), de 1,3 a 1,9 g /MS/ bocado/animal. Em experimento com vacas leiteiras adultas, Hodgson (1990) reportou valores de 0,2 a 1,0 g e Barrett et al. (2003), de 0,7 a 1,2 g MS/bocado/vaca.

Como resposta aos valores crescentes de massa do bocado, a taxa de bocados praticada pelos animais reduziu linearmente, de 31 para 15 bocados/minuto entre a menor e a maior altura testadas (Figura 1c).

Esses valores foram inferiores ao de 55 bocados/minuto reportado por Silva et al. (2003) em trabalho com novilhos de corte em pastagens de clima temperado e ao de 30 a 70 bocados/ minuto sugerido por Carvalho (1997) para herbívoros em geral. Barrett et al. (2003), testando genótipos de azevém, registraram 47 a 56 bocados/minuto quando as plantas se encontravam nos estádios vegetativo e reprodutivo, respectivamente.

Em pastagens de espécies cespitosas como capimmarandu (Brachiaria brizantha, Hochst. ex A. Rich.) Stapf, Santos et al. (2003) registraram para novilhas Nelore amplitude de 17,5 a 46,3 bocados/minuto, valores semelhantes aos obtidos neste trabalho. Taxas de bocado muito baixas em pastagens de gramíneas tropicais cespitosas foram reportadas por Carvalho et al (2001a) e Castro (2002), indicando que esse tipo de estrutura limitaria o processo de ingestão de forragem.
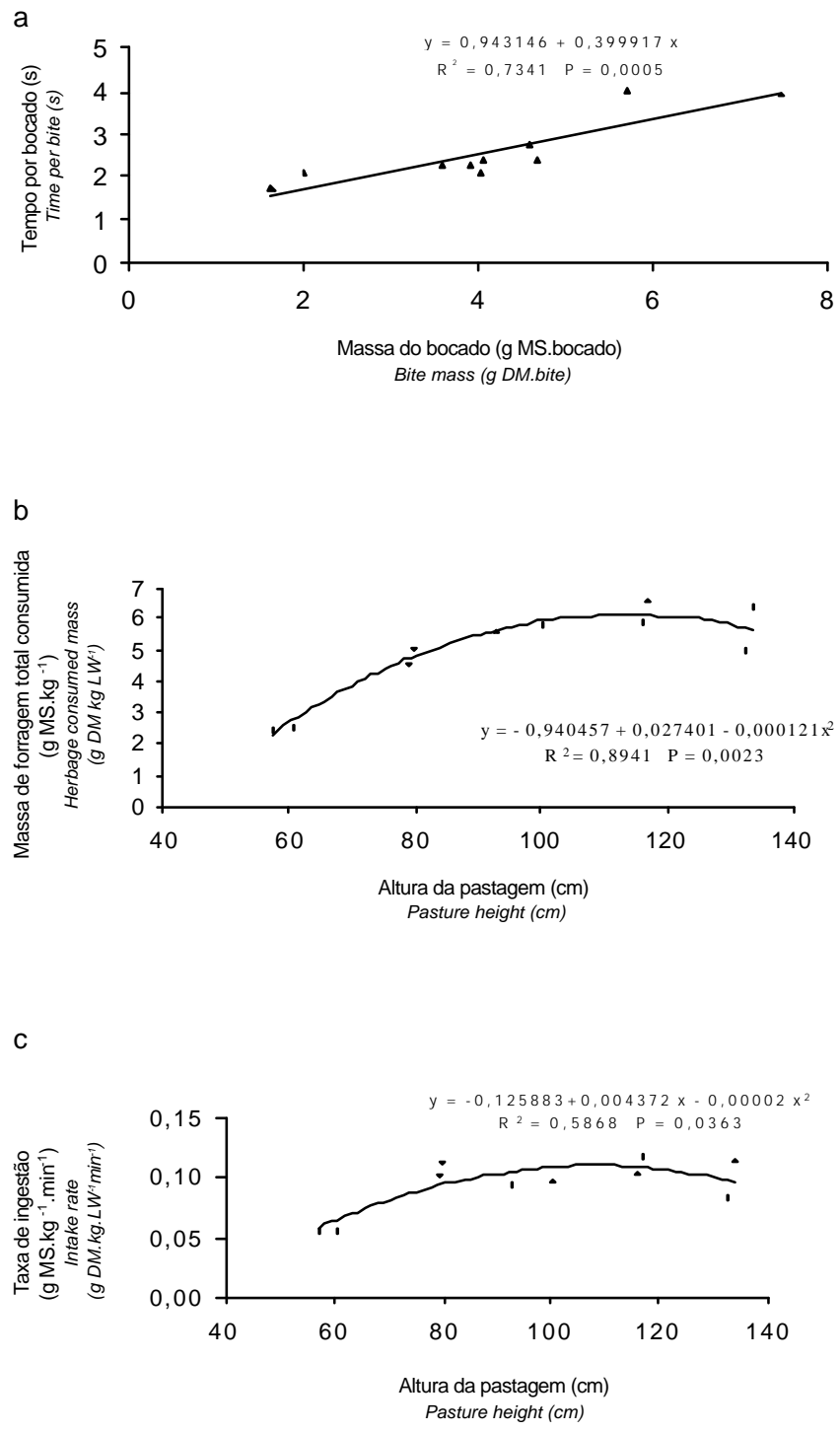

d

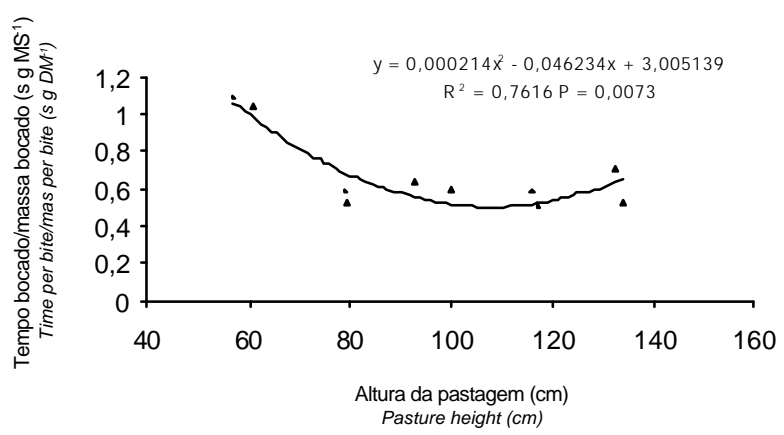

Figura 2 - a) Relação massa e tempo por bocado (g MS s ${ }^{-1}$ ); relação altura da pastagem e b) massa seca total consumida (g MS $100 \mathrm{~kg}^{-1}$ ), c) taxa de ingestão (g MS min $^{-1}$ ) e d) tempo por bocado/massa do bocado (s $\mathrm{g} \mathrm{MS}^{-1}$ ) de novilhas leiteiras em pastagem de capim-mombaça.

Figure 2 - a) Relationship between mass and time per bite ( $\left.g D M s^{1}\right)$; relationship between sward height and $b$ ) total mass consumed $(g D M), c)$ intake rate and d) time per bite/bite mass (s $g$ MS ${ }^{-1}$ ) by Holstein heifers in mombaçagrass pasture. 
A redução na taxa de bocados com o aumento da altura do dossel também foi registrada por Griffiths et al.(2003)e pode ser explicada pelo aumento significativo no tempo de formação de bocados maiores, que variou de 1,95 a 3,99 segundos neste experimento (Figura 1d), ou seja, houve acréscimo de $100 \%$ no tempo para realização de cada bocado entre a menor e a maior altura avaliada, incremento superior ao de $40 \%$ verificado por Carvalho et al. (2001a), em pastagem de capim-tanzânia pastejada por ovinos.

Essa situação sugere que o animal gastou mais tempo no processo de apreensão das lâminas foliares à medida que essas lâminas aumentaram em comprimento em relação à altura do dossel (Figura 1e). As folhas passaram a ser ingeridas uma a uma, estabelecendo o que Carvalho et al. (2001a) classificaram como pastejo "tipo espaguete". A menor eficiência dos movimentos de apreensão com a distribuição esparsa da forragem nos estratos superiores e com o elevado comprimento das lâminas foliares presentes nos estratos superiores do relvado resultou em aumento no tempo de formação dos bocados.

Além disso, é provável que o aumento na massa ingerida, decorrente do maior teor de MS e do maior teor de fibra nessa MS, tenha exigido maior processamento pelos animais (Penning, 1986; Prache \& Peyraud, 2001), o que aumentou o tempo e o número necessário de movimentos de mastigação (Van Vuuren, 1994; Tharamaj et al, 2003).

Na Figura 2a consta a relação linear e positiva entre massa do bocado e tempo por bocado. O valor da intercepta, aproximadamente 1 , refere-se ao tempo necessário à abertura e ao fechamento da boca do animal durante a formação de cada bocado, considerado "custo fixo" para o processo de apreensão de forragem. Nas menores alturas do dossel, considerando que a massa do bocado apresentou valores menores (Figura 1b), os animais compensaram a menor ingestão com o aumento no número de movimentos de apreensão (Prache $\&$ Peyraud, 2001), visto que a apreensibilidade da forragem e as exigências de mastigação reduziram, como resultado da menor massa ingerida em cada bocado.

Outro fator que pode ter contribuído para o aumento no tempo de formação de cada bocado foi o aumento nos teores de FDN e FDA com o incremento na altura do dossel (Tabela 1), o que deve ter dificultado a apreensão da forragem. A fibrosidade da forragem, passível de predição a partir de parâmetros como a FDN (Prache \& Peyraud, 2001), pode dificultar a ruptura dos tecidos vegetais (Van Vuuren, 1994) e aumentar o tempo para mastigação da forragem (Illius et al., 1995), interferindo no tempo final de formação de cada bocado. Segundo Prache \& Peyraud (2001), a apreensibilidade da forragem pode apresentar comportamento semelhante ao da digestibilidade, diminu- indo com a idade da planta e/ou com a profundidade do relvado (Tharmaraj et al., 2003). No entanto, segundo esses autores, a relação custo:benefício relacionada à apreensão de forragem é sempre positiva e, portanto, o processo de apreensão não parece ser limitado pelo custo energético envolvido nessa atividade.

O aumento na taxa de bocados e/ou no tempo de pastejo com objetivo de compensar a redução na massa do bocado em condições de pastejo e oferta restrita (dosséis baixos) é amplamente descrito na literatura (Arnold, 1987; Hodgson, 1990; Carvalho et al., 2001a) e faz parte das estratégias de forrageamento dos animais (Gordon \& Illius, 1992). No entanto, essa estratégia pode não ser suficiente e ocasionar a redução na ingestão de forragem, em virtude da flexibilidade do animal em aumentar o tempo de alimentação, limitada à medida que o animal executa ao longo do dia outras atividades como ruminação, descanso e atividades sociais (Carvalho et al, 2001b).

Em condições de clima temperado, os modelos de predição de ingestão consideram constante o tempo de apreensão da forragem pelos animais (Carvalho et al., 2001 b). Porém, como comprovam os resultados obtidos neste experimento e aqueles relatados por Carvalho et al. (2001 a) em condições tropicais, o tempo de formação dos bocados é variável e s ofre influência marcante da estrutura do dossel forrageiro.

A baixa densidade volumétrica da forragem nos estratos superiores do dossel (Tabela 2) não limitou a massa do bocado, que aumentou linearmente de acordo com a altura do dossel (Figura 1b), determinando padrão análogo de resposta, como descrito por Stobbs (1973a,b). Apesar desse aumento, a massa de forragem total consumida e a taxa de ingestão sofreram tendência quadrática, com os valores praticamente estáveis nas maiores alturas de dossel (Figuras 2b e c).

Os valores observados para a taxa de ingestão variaram de 7,5 a 17,0 g MS/ minuto/animal entre a menor e a maior altura do dossel e foram inferiores aos descritos por Damasceno et al. (2003a), que reportaram valores de 19,0 a 38,0 g MS/minuto para novilhos em pastagem de capim-tanzânia. A estabilização da massa de forragem total consumida e da taxa de ingestão nas maiores alturas do dossel pode ser explicada pelo acréscimo proporcionalmente maior no tempo necessário à formação de cada bocado, comparativamente ao acréscimo observado para a massa de bocado, notadamente nas duas maiores alturas do dossel estudadas.

Com o incremento na altura do dossel, o tempo de formação dos bocados aumentou $66,9 \%$ e a massa do bocado $51,2 \%$ entre as alturas de 120 e 140 cm, ocasionando redução significativa na taxa de bocados e limitando a taxa de ingestão, indicando possível decréscimo no consumo na altura de $140 \mathrm{~cm}$. Esse fato aponta para uma eficiência crescente de 
captura da forragem com o aumento na altura do dossel até $100 \mathrm{~cm}$, quando estabilizou e passou a decrescer (Figura 2c).

Padrão semelhante de resposta foi reportado por Carvalho et al. (2001a), que observaram redução na taxa de consumo de ovinos decorrente do maior tempo necessário à formação do bocado, como conseqüência da disposição esparsa das folhas do capim-tanzânia nos estratos mais elevados do dossel. Peyraud et al. (1995), avaliando ofertas de forragem em espécies de clima temperado, verificaram estabilização da taxa de bocados e da taxa de ingestão como resultado da necessidade de manipulação excessivamente longa da forragem nas maiores ofertas de forragem.

Nesse contexto, mesmo que os aumentos no tempo para formação de bocados sejam reduzidos, o elevado número de bocados ao longo do dia pode conduzir a um tempo de pastejo excessivamente longo (Carvalho et al., 2001b; Tharmaraj et al., 2003), o que pode ser prejudicial aos animais, pois existem outras atividades igualmente essenciais e que podem ter seu tempo significativamente reduzido.

Uma vez que os testes de pastejo aplicados foram de curta duração, em virtude das características específicas da hipótese estudada, não foi possível avaliar se os animais aumentaram o tempo de pastejo ao longo do dia visando compensar a menor ingestão de forragem durante o período de aplicação dos testes nas maiores alturas de dossel estudadas. Essa extrapolação é difícil, pois, segundo Prache et al. (1998), as estratégias alimentares dos animais utilizadas em curto prazo podem não ser previsíveis quando considerados os aspectos do comportamento ingestivo em longo prazo, situação em que a complexidade dos fatores envolvidos é maior. Ao integrar os efeitos dos processos ingestivos e digestivos, o tempo de pastejo caracteriza-se por ser uma variável particularmente complexa e de difícil entendimento, como reportado por Carvalho (1997) ao classificá-la como a "caixa preta" do processo de pastejo, o que justifica a necessidade de pesquisa e elucidação dos mecanismos que atuam sobre essa variável.

Os resultados deste estudo estão de acordo com os descritos por Ungar (1996) e Hodgson et al (1994), que sugeriram que o número de movimentos de manipulação está mais relacionado à estrutura do dossel que à massa do bocado. No entanto, os resultados obtidos em pastagens de clima temperado não evidenciam (provavelmente pela limitada amplitude de variação estudada para essa característica) a magnitude do impacto da estrutura do dossel sobre o processo de ingestão. A possibilidade de haver restrição ao consumo em elevadas ofertas de forragem por processos não associados à composição química da forragem traz novas e importantes hipóteses para o manejo de pastagens tropicais, com evidentes implicações a sistemas de pastejo orientados para máximo acúmulo de forragem. Por exemplo, o uso de pastejo rotacionado em situações de elevado período de descanso e/ou o uso de doses elevadas de fertilizantes podem produzir freqüentemente estruturas que nem sempre priorizam a interceptação de radiação e o acúmulo líquido de lâminas foliares (Carnevalli et al., 2006), e que tampouco priorizam a captura da forragem pelo animal em pastejo.Uma vez que a maioria dos sistemas de produção não comercializa forragem, mas sim o produto animal, o manejo da pastagem deve priorizar o oferecimento de forragem em estrutura conveniente à otimização do processo de captura pelo pastejo em vez de focar apenas a produção de forragem, como visto freqüentemente na literatura.

\section{Conclusões}

O comportamento ingestivo de animais em pastejo é sensível a variações na estrutura do dossel forrageiro.

Em condições de elevada oferta de forragem, a eficiência do processo de pastejo pode ser limitada pela dificuldade de apreensão das lâminas foliares.

Os resultados apontam para a necessidade de estudar o manejo da pastagem como a construção de ambientes pastoris mais adequados à otimização do processo ingestivo.

\section{Literatura Citada}

ARNOLD, G.W. Grazing behavior. In: SNAYDON, R.W. (Ed.) Managed grassland analytical studies. Amsterdam: Elsevier, 1987. p.129-135.

BARRETT, P.D.; McGILLOWAY, D.A.; LAIDLAW et al. The effect of sward structure as influenced by ryegrass genotype on bite dimensions and short term intake rate by dairy cows. Grass and Forage Science, v.58, p.2-11, 2003.

BARTHRAM, G.T. Experimental techniques: the HFRO sward stick. Hill Farming Research Organization/Biennial Report. 1985. p.29-30.

BURNS, J.C.; LIPPKE, H.; FISHER, D.S. The relationship of herbage mass and characteristics to animal responses in grazing experiments. In: MARTEN, G.C. (Ed.) Grazing research: design, methodology and analysis. Madison: Crop Science Society of America, 1989. p.7-20.

CANGIANO, C.A.; GALLI, J.R.; PECE, M.A. et al. Effect of live weight and pasture height on cattle bite dimensions during progressive defoliation. Australian Journal of Agricultural Research, v.53, p.541-549, 2002.

CARNEVALLI, R.A.; DA SILVA, S.C.; BUENO, A.A.O. et al. Herbage production and grazing losses in Panicum maximum cv. Mombaça under four grazing managements. Tropical Grasslands, v.40, n.3, p.165-176, 2006.

CARVALHO, P.C.F. A estrutura da pastagem e o comportamento ingestivo de ruminantes em pastejo In: SIMPÓSIO SOBRE AVALIAÇÃO DE PASTAGENS COM ANIMAIS, 2., 1997, Maringá. Anais... Maringá: Universidade Estadual de Maringá, 1997. p.25-52.

CARVALHO, P.C.F.; MARÇAL, G.K.; RIBEIRO FILHO, H.M.N. et al. Pastagens altas podem limitar o consumo dos animais. In: REUNIÃO ANUAL DA SOCIEDADE BRASILEIRA DE ZOOTECNIA, 38., 2001, Piracicaba. Anais... Piracicaba: Escola Superior de Agricultura "Luiz de Queiroz", 2001a. p.265-268. 
CARVALHO, P.C.F.; RIBEIRO FILHO, H.M.N.; POLI, C.H.E.C.et al. Importância da estrutura da pastagem na ingestão e seleção de dietas pelo animal em pastejo. In: PEDREIRA, C.G.S.; DA SILVA, S.C. (Eds.) A produção animal na visão dos brasileiros. Piracicaba: Fundação de Estudos Agrários Luiz de Queiroz, 2001b. p.853-871.

CASTRO, C.R.C. Relações planta-animal em pastagem de milheto (Pennisetum clandestinum (L.) Leeke) manejadas em diferentes alturas com ovinos. Porto Alegre: Universidade Federal do Rio Grande do Sul, 2002. 200p. Dissertação (Mestrado em Zootecnia, Plantas Forrageiras) - Universidade Federal do Rio Grande do Sul, 2002.

DAMASCENO, J.C.; REGO, F.L.A.; CORTES, C. et al. Pasture intake rate by steers in response to sward structure. In: WORLD CONFERENCE ON ANIMAL PRODUCTION, 9., 2003, Porto Alegre. Proceedings... Porto Alegre: Universidade Federal do Rio Grande do Sul, 2003a. p.120.

DAMASCENO, J.C.; REGO, F.L.A.; FROHMUT, K.R. et al. Bite characteristics in response to intake rate by steers and sward structure in Panicum maximum (Tanzânia grass), Brachiaria brizantha and Arachis pintoii pastures. In: WORLD CONFERENCE ON ANIMAL PRODUCTION, 9., 2003, Porto Alegre. Proceedings... Porto Alegre: Universidade Federal do Rio Grande do Sul, 2003b. p.120.

DEMMENT, M.W.; LACA, E.A The grazing ruminant: models and experimental techniques to relate sward structure and intake. In: WORLD CONFERENCE ON ANIMAL PRODUCTION, 7., 1993, Edmonton. Proceedings... Edmonton: 1993. p.439-460.

DURU, M.; DUCROCQ, H.; BOSSUET, L. Herbage volume per animal: a tool for rotational grazing management. Journal of Range Management, v.53, n.4, p.395-402, 2000.

GORDON, I.J.; ILLIUS, A.W. Foraging strategies: from monocultures to mosaics. In: SPEEDY, A.W. (Ed.) Progress in sheep and goat research. Wallingford: $C A B$ International, 1992. p.153-178.

GORDON, I.J.; LASCANO, C. Foraging strategies of ruminant livestock on intensively managed grasslands: potential and constraints. In: INTERNATIONAL GRASSLAND CONGRESS, 17., 1993, Palmerston North. Proceedings... Palmerston North: 1993. p.681-690.

GRIFFITHS, W.M.; HODGSON, J.; ARNOLD, G.C. The influence of sward canopy structure on foraging decisions by grazing cattle. I. Patch selection. Grass and Forage Science, v.58, p.112124, 2003.

HODGSON, J. Grazing management: science into pratice. New York: John Wiley; Longman Scientific and Technical, 1990. 203p.

HODGSON, J.; CLARK, D.A; MITCHELL, R.J. Foraging behavior in grazing animals and its impact on plant communities. In: FAHEY, G.C.; COLLINS, M.; MERTENS, D.R. et al. (Eds.) Forage quality, evaluation and utilization. Lincoln: American Society of Agronomy. 1994. p.796-827.

ILLIUS, A.W.; GORDON, I.J.; MILNE, J.D., WRIGHT, W. Costs and benefits of foraging on grasses varying in canopy structure and resistance to defoliation. Functional Ecology, v.9, p.894903, 1995.

ILLIUS, A.W.; GORDON, I.J. The physiological ecology of mammalian herbivory. In: INTERNATIONAL SYMPOSIUM ON THE NUTRITION OF HERBIVORES, 6., 1999, Savoy. Proceedings... Savoy: 1999. p.407-423.

LACA, E.; DEMMENT, M.W. Herbivory: the dilemma of foraging in spatially heterogeneous food enviroment. In: PALO, R.T., ROBINS C.T. (Ed.) Plant Defenses against mammalian herbivores. Boca Raton: 1991. p.29-44.

LEMAIRE, G.; GASTAL, F. Nitrogen uptake and distribution in plant canopies. In: LEMAIRE, G. (Ed.) Diagnosis of the nitrogen status in crops. Berlin: Sperlinger Verlag Berlin Heidelberg, 1997. p.3-43.

MILNE, J.A. Diet selection by grazing animals. In: NUTRITION SOCIETY, 50., 1991, Oxford. Proceedings... Oxford: 1991. p.77-85.
NABINGER, C.; PONTES, L.S. Morfogênese de plantas forrageiras e estrutura do pasto. In: PEDREIRA, C.G.S.; DA SILVA, S.C. (Eds.) A produção animal na visão dos brasileiros. Piracicaba: Fundação de Estudos Agrários Luiz de Queiroz, 2001. p.755-771.

PENNING, P.D. Some effects of sward conditions on grazing behavior and intake by sheep. In: GUNDMUNDSSON, O. (Ed.) Grazing research at northern latitudes. Hvanneyri: NATO Advanced Research Workshop, 1986. v.40, p.79-84.

PENNING, P.D.; HOOPER, G.E.N. A evaluation of the use of shortterm weight changes in grazing sheep for estimating herbage intake. Grass and Forage Science, v.40, p.79-84, 1985.

PENNING, P.D.; PARSONS, A.J.; ORR, R.J. Intake and behavior responses by sheep to changes in sward characteristics under continuous stocking. Grass and Forage Science, v.46, p.1528, 1991.

PEYRAUD, J.L.; DELAGARDE, R.; DELABY, L. Influence des conditions d'exploitation du pâturage et des caractéristiques animales sur les quanitités ingérées par les vaches laitières: analyse et prédiction. Rencontres Recherches Ruminantes, v.2, p.37-44, 1995

PRACHE, S.; GORDON, I.J.; ROOK, A.J. Foraging behaviour and diet selection in domestic herbivores. Annales de Zootechnie, v.48, p.1-11, 1998.

PRACHE, S.; PEYRAUD, J. Foraging: behaviour and intake in temperate cultivated grassland. In: INTERNATIONAL GRASSLAND CONGRESS, 19., 2001, São Pedro. Proceedings... São Pedro: 2001. p.309-319.

SANTOS, I.B.; SIQUEIRA, A.K.R.; REVIGLIO, I.L. et al. Taxa de bocados de bovinos de corte em capim-marandu submetidos a regimes de lotação contínua. In: SIMPÓSIO INTERNACIONAL DE INICIAÇÃO CIENTÍFICA DA USP (SIICUSP), 11., 2003, Piracicaba. Anais... Piracicaba: Escola Superior de Agricultura Luiz de Queiroz, 2003. (CD-ROM).

SILVA, A.C.F.; QUADROS, F.L.F.; TREVISAN, N.B. et al. Comportamento ingestivo e taxa de bocados de terneiros de corte em pastagem de estação fria sob diferentes níveis de biomassa de lâmina foliar verde. In: REUNIÃO ANUAL DA SOCIEDADE BRASILEIRA DE ZOOTECNIA, 40., 2003, Santa Maria. Anais... Santa Maria: Universidade Federal de Santa Maria, 2003. (CD-ROM).

STOBBS, T.H. The effects of plant structure on the intake of tropical pastures. I. Variation in the bite size of grazing cattle. Australian Journal of Agricultural Research, v.24, p.809819, 1973a.

STOBBS, T.H. The effects of plant structure on the intake of tropical pastures. II. Diferences in sward structure, nutritive value and bite size of animals grazing Setaria anceps and Chloris gayana at various stages of growth. Australian Journal of Agricultural Research, v.24, p.821-829, 1973b.

THARMARAJ, J.; WALES, W.J.; CHAPMAN, D.F. et al. Defoliation pattern, foraging behaviour and diet selection by lactating dairy cows in response to sward height and herbage allowance of a rye-grass dominated pasture. Grass and Forage Science, v.98, p.225-238, 2003.

UNGAR, E.D. Ingestive behaviour. In: HODGSON, J., ILLIUS, A. (Eds.) The ecology and management of grazing systems. Wallingford: CAB International, 1996. p.185-218.

Van SOEST, P.J. Enviroment and forage quality. In: CORNELL NUTRITION CONFERENCE FOR FOOD MANUFACTURERS, 58., 1996, Ithaca. Proceedings... Ithaca: Cornell University, 1996. p.1-9.

Van VUUREN, A.M. Aspects of forage intake regulation. In: GENERAL MEETING OF THE EUROPEAN GRASSLAND FEDERATION, 15., 1994, Wageningen. Proceedings... Wageningen: 1994. p.556-565.

Recebido: 23/06/05 Aprovado: 27/02/07 